\title{
Recent developments of quantum dot based micro-LED based on non-radiative energy transfer mechanism
}

\author{
Xiaotong $\mathrm{Fan}^{1 \dagger}$, Tingzhu $\mathrm{Wu}^{1,2 \dagger}$, Bin $\mathrm{Liu}^{3}$, Rong Zhang ${ }^{1,3}$, \\ Hao-Chung $\mathrm{Kuo}^{4 *}$ and Zhong Chen ${ }^{1,2 *}$
}

With regard to micro-light-emitting diodes (micro-LEDs), their excellent brightness, low energy consumption, and ultrahigh resolution are significant advantages. However, the large size of traditional inorganic phosphors and the number of side defects have restricted the practical applications of small sized micro-LEDs. Recently, quantum dot (QD) and nonradiative energy transfer (NRET) have been proposed to solve existing problems. QDs possess nanoscale dimensions and high luminous efficiency, and they are suitable for NRET because they are able to nearly contact the micro-LED chip. The NRET between QDs and micro-LED chip further improves the color conversion efficiency (CCE) and effective quantum yield (EQY) of full-color micro-LED devices. In this review, we discuss the NRET mechanism for QD micro-LED devices, and then nano-pillar LED, nano-hole LED, and nano-ring LED are introduced in detail. These structures are beneficial to the NRET between QD and micro-LED, especially nano-ring LED. Finally, the challenges and future envisions have also been described.

Keywords: quantum dot based micro-LED; non-radiative energy transfer; atomic layer deposition; sidewall defects

Fan XT, Wu TZ, Liu B, Zhang R, Kuo HC et al. Recent developments of quantum dot based micro-LED based on non-radiative energy transfer mechanism. Opto-Electron Adv 4, 210022 (2021).

\section{Introduction}

Display technology has gone through countless changes and penetrated every corner of our life. As a display technology, light-emitting diode (LED) has attracted attention due to its low cost, easy fabrication, and energy conservation $^{1,2}$. In 2000, the technology strategy of microLEDs was put forward for the first time at the Texas Tech University, which signified that LED light sources had entered the era of micro display ${ }^{3-5}$. Compared with traditional LED screen display technologies such as miniLED, organic-LED, etc., the micro-LEDs have the following advantages: high brightness, high luminous efficiency, low energy consumption, quick reaction, high contrast, self-illumination, long service life, ultra-high resolution, and good color saturation ${ }^{6-9}$.

The RGB full-color display has been employed

'School of Electronic Science and Engineering, Fujian Engineering Research Center for Solid-State Lighting, Xiamen University, Xiamen 361005, China; ${ }^{2}$ Fujian Science \& Technology Innovation Laboratory for Energy Materials of China, Xiamen 361005, China; ${ }^{3}$ Jiangsu Provincial Key Laboratory of Advanced Photonic and Electronic Materials, Nanjing National Laboratory of Microstructures, School of Electronic Science and Engineering, Nanjing University, Nanjing 210093, China; ${ }^{4}$ Department of Photonics and Graduate Institute of Electro-Optical Engineering, College of Electrical and Computer Engineering, Chiao Tung University, Hsinchu 30010, China.

tThese authors contributed equally to this work.

"Correspondence: HC Kuo, E-mail: hckuo@faculty.nctu.edu.tw; Z Chen, E-mail: chenz@xmu.edu.cn

Received: 14 February 2021; Accepted: 12 March 2021; Published: 6 April 2021

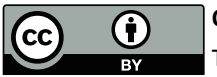

Open Access This article is licensed under a Creative Commons Attribution 4.0 International License.

To view a copy of this license, visit http://creativecommons.org/licenses/by/4.0/.

(C) The Author(s) 2021. Published by Institute of Optics and Electronics, Chinese Academy of Sciences. 
widely $^{10-13}$. In this method, different currents are applied to control the brightness of each LED to realize the combination of three primary colors within a full-color display. In the RGB full-color display method, each pixel contains a set of RGB LEDs.

However, technology based on RGB micro-LEDs has serious disadvantages in mass production. For example, to make $4 \mathrm{~K}$ resolution displays, it is necessary to assemble and drive nearly 25 million micro-LEDs in an economical and efficient way, with a placement accuracy of less than $1 \mu \mathrm{m}$. It is very difficult to transfer or grow three different micro-LEDs in such large quantities on the same substrate. As shown in Fig. 1(a), several methods including laser-induced forward transfer and anisotropic conductive film bonding are utilized to help mass transfer ${ }^{14,15}$. Apple, Samsung, Sony, and other manufacturers are actively studying to improve the yield and efficiency in massive transfer technology, and although they have made some progress, the current production cost is still severe.

To avoid the use of mass transfers, the color conversion scheme of quantum dot (QD) has been proposed $^{16,17}$, which is based on the ability of QDs to realize RGB full-color displays under the excitation of blue light or ultraviolet micro-LED chips ${ }^{18-20}$. QDs are nanomaterials composed of a small number of atoms, which restrict the electron movement in three-dimensional space ${ }^{21}$. When the size of crystal is smaller than the exciton Bohr radius (usually less than $50 \mathrm{~nm}$ ), the energy levels of the crystal are discrete and the band gap is dependent on the physical size. Therefore, adjusting the size of nanoparticle (QD) can easily achieve luminescence with different wavelengths. As the color conversion materials of micro-LED, QDs could replace traditional phosphors, which are difficult to apply due to their large size. Further, as shown in Fig. 1(a), because QDs with different luminescence colors only need one type of excitation chip, a large amount of the transfer

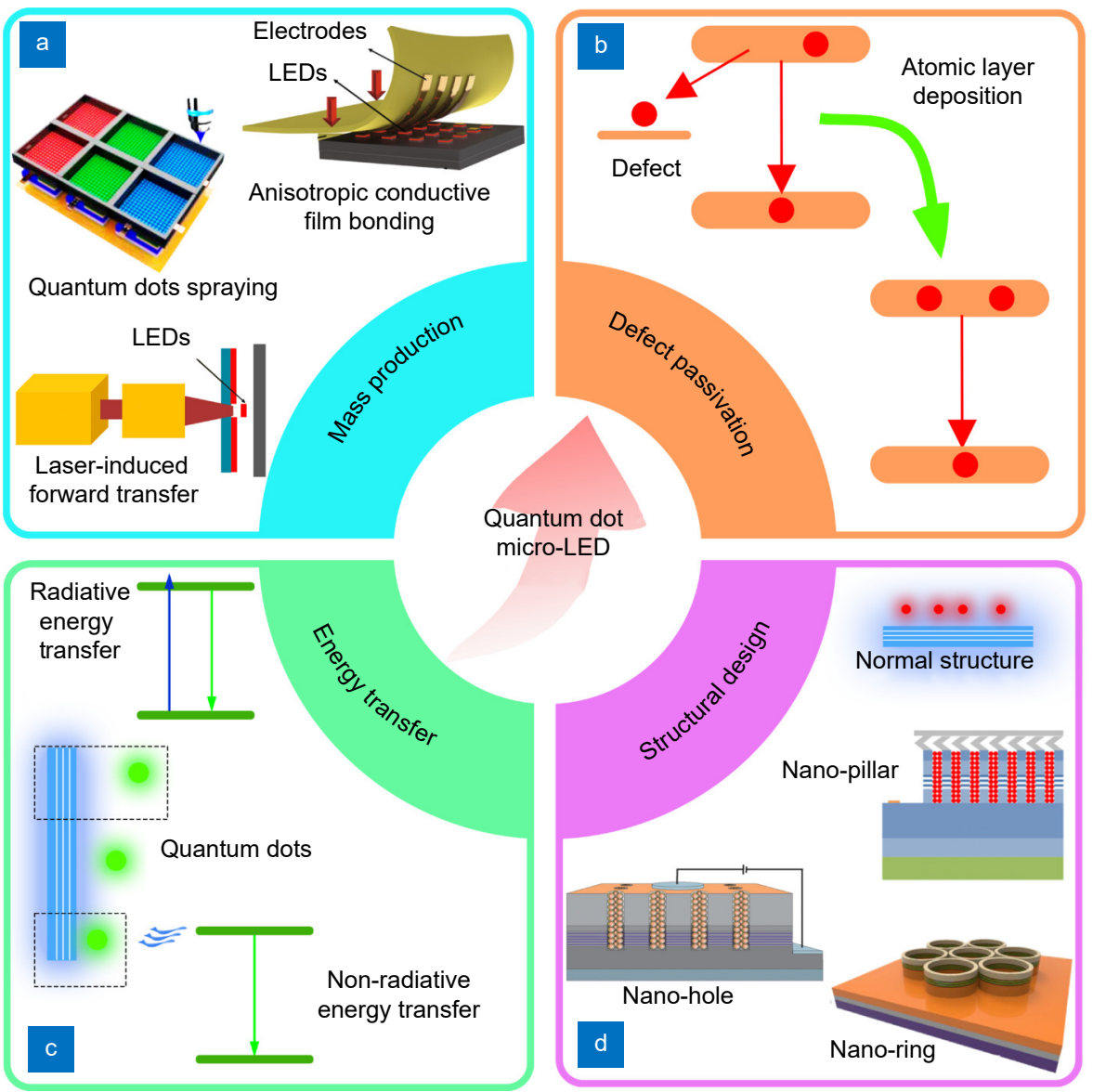

Fig. 1 The corresponding developments of QD micro-LED. (a) The methods of mass production. (b) Atomic layer deposition passivation. (c) The mechanism of energy transfer between micro-LED chip and QDs. (d) Structural design for QD micro-LEDs. Figures reproduced with permission from: (a) ref. ${ }^{14}$, Royal Society of Chemistry; ref. ${ }^{15}$, under a Creative Commons Attribution License; ref. ${ }^{22}$, Chinese Laser Press; (d) ref. ${ }^{28}$, Optical Society of America; ref. ${ }^{29}$, John Wiley and Sons; ref. ${ }^{30}$, under a Creative Commons Attribution License. 
preparation process can be avoided during QDs spraying method $^{22}$, which gives the QD micro-LED full-color display scheme an excellent commercial potential.

Through hybridization of different QD combinations, it is quite convenient to adjust white light parameters, including the chromaticity coordinates, correlated color temperature, and color rendering index. A hybrid white LED using QDs as down-conversion materials was previously demonstrated by Chen et al. ${ }^{23}$, exhibiting a high color rendering index of 91 and a CIE-1931 coordinate of $(0.33,0.33)$.

To achieve excellent luminous properties, it is very important to realize an efficient combination and energy transfer of chips and emitters. Sidewall defects seriously restrict the transfer between micro-LED and QDs, hence, defect passivation of micro-LED is necessary, as shown in Fig. 1(b), atomic layer deposition (ALD) as a suitable method has been reported ${ }^{24}$. However, common devices are based on blue/ultraviolet chips that radiatively pump blue, green, and red-emitting QDs with a low color conversion efficiency (CCE), resulting from the large separation between the active layer in the LEDs and QDs. To overcome this drawback, non-radiative energy transfer (NRET) was proposed as another method for color conversion, as shown in Fig. $1(\mathrm{c})^{25-27}$. The close contact between the chip and the emitter is a key factor in realizing NRET, so the design of micro-LED structures, such as nano-pillar ${ }^{28}$, nano-hole ${ }^{29}$, and nano-ring ${ }^{30}$ as shown in Fig. 1(d), is also required. In this article, we review recent researches on the application of the NRET mechanism in micro-LEDs with various structures and put forward some helpful suggestions for existing issues.

\section{Non-radiative energy transfer}

Unlike traditional fluorescence conversion, the NRET mechanism is realized through the coupling between dipoles. This function is directly completed at one time through the non-radiative resonance form and does not involve the absorption and conversion of photons. The reemission process reduces the energy loss caused by these indirect processes and can significantly improve the CCE even quantum yield (QY) $)^{31-34}$. We listed several studies and compared the luminous performance of LED device based mainly radiative energy transfer or NRET, the results are summarized in Table 1.

The NRET mechanism mainly depends on three conditions $^{38,39}$ : that the degree of overlap between the emission wavelength of the donor and the absorption wavelength of the acceptor is large, that the directions between dipoles need to be parallel, and that the distance from the donor to the acceptor is small enough (less than $10 \mathrm{~nm}$ ). Therefore, to utilize the NRET mechanism to improve the CCE of the system, the spatial distance between the QD and the active area of the microLED chip must be as small as possible.

Achermann et al. discussed the NRET mechanism between semiconductors with an epitaxial quantum well $(\mathrm{QW})^{35}$. The structure of the device in their work included InGaN QW, a GaN cap with a thickness of $3 \mathrm{~nm}$, and CdSe/ZnS core/shell QDs. The emission wavelength of InGaN was between 370 and $450 \mathrm{~nm}$, while the absorption wavelength of CdSe/ZnS QDs ranged from 380 to $600 \mathrm{~nm}$, as shown in Fig. 2. The short distance and overlap of the emission wavelength of the donor (In$\mathrm{GaN}$ ) with the absorption of the acceptor (QDs) were vital for NRET.

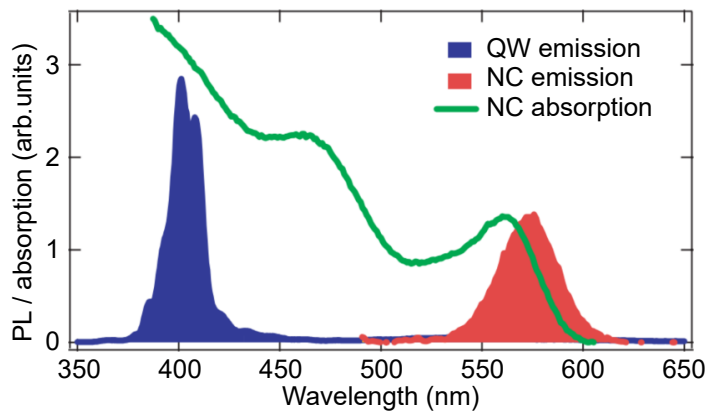

Fig. 2 | Optical properties of the hybrid QW/QDs. Figure reproduced with permission from ref. ${ }^{35}$, Springer Nature.

To directly prove the NRET process, the time-resolved photoluminescence (TRPL) measurements are essential. The authors tested the change in the delay time

Table 1 | Luminous performance of LED device based mainly radiative energy transfer or NRET.

\begin{tabular}{|c|c|c|c|}
\hline Reference & Performance & Radiative energy transfer & Non-radiative energy transfer \\
\hline Ref. ${ }^{33}$ & Quantum yield & $55 \%$ & $95 \%$ \\
\hline Ref. ${ }^{35}$ & Quantum yield & $20 \%$ & $55 \%$ \\
\hline Ref. ${ }^{36}$ & Color conversion efficiency & $3.5 \%$ & $13 \%$ \\
\hline Ref. $^{37}$ & Effective quantum yield & $56.0 \%$ & $81.2 \%$ \\
\hline
\end{tabular}


of QW under different conditions. The QW delay shortened with the increase in the QD content, indicating an additional relaxation channel for QW excitation, which was believed to have been rooted out of NRET from QW to QDs. More evidence for efficient NRET from QW to QDs was provided by the photoluminescence intensity of QDs. At the same excitation density, the QDs with QW had higher intensity, indicating that NRET promoted an increase in luminescence.

Researchers have proposed a schematic of NRET along with other relaxation processes in the QW/QD structure. After excitation, carrier thermalization, and cooling, the thermal distribution of free electrons and holes was established in QW. QW carriers could decay either radiatively (time constant $\tau_{\mathrm{rr}}$ ), non-radiatively $\left(\tau_{\mathrm{nr}}\right)$, or experience energy transfer $\left(\tau_{\mathrm{ET}}\right)$ into QDs. Excitons generated in the QDs by NRET had significant access energies as measured with respect to the bandgap of QDs. In QDs, extremely fast band relaxation (sub-picosecond time scales) can quickly eliminate the resonance between carriers and QW transitions and prevent reverse transfer. In the well-passivated QDs, the relaxed electron hole pairs were mainly recombined by radiation with a time constant of $\sim 20$ ns. The non-radiative energy transfer efficiency from QW to QDs could be estimated using the following expression:

$$
\begin{aligned}
& \eta=\tau_{\mathrm{r}}\left(\tau_{\mathrm{ET}}+\frac{1}{\tau_{\mathrm{r}}}\right)^{-1}, \\
& \tau_{\mathrm{r}}=\left(\frac{1}{\tau_{\mathrm{rr}}}+\frac{1}{\tau_{\mathrm{nr}}}\right)^{-1},
\end{aligned}
$$

where $\tau_{\mathrm{r}}$ is the relaxation time of the QW excitation caused by radiative and non-radiative processes.

Several studies have also reported that NRET improves the luminescence quality of QDs. Achermann et al. discussed the CCE with NRET in another development ${ }^{36}$. CdSe/ZnS core/shell QDs were usually dissolved in toluene and spin-coated on the n-type layer of GaNbased LEDs in the form of a monolayer. However, according to the inter-QD interactions, which could lead to exciton transfer from the emitter to near QDs, the photoluminescence quantum yield (PLQY) decreased from $60 \%$ in the solution to $35 \%$ in the monolayer. The author chose 35\% to calculate the CCE of the QDs. In their study, the CCE is calculated according to the simplified expression:

$$
\begin{gathered}
C C E \approx P L Q Y_{\mathrm{QD}} \frac{k_{\mathrm{ET}}}{k_{\mathrm{QW}}^{\mathrm{rad}}}, \\
P L Q Y_{\mathrm{QD}}=\frac{E_{\mathrm{em}}}{E_{\mathrm{abs}}},
\end{gathered}
$$

where $k_{\mathrm{ET}}$ is the theoretical energy transfer rate between $\mathrm{QW}$ and QDs, $k_{\mathrm{QW}}^{\mathrm{rad}}$ is the decay rate for nonradiative in the $\mathrm{QW}^{32}$. The $E_{\mathrm{abs}}$ and $E_{\mathrm{em}}$ are the absorbed photons energy and the emitted photons energy, respectively.

They estimated that CdSe/ZnS core/shell QDs excited by photoluminescence modes could result in approximately 3.5\% CCE. However, the measured CCE was 13\% when QDs were excited through a mechanism based on absorption-reemission. Taking into account the potential contribution from absorption remission, the CCE produced by the NRET process in the single-QDs-layer structures could be estimated at approximately $10 \%$.

NRET is also expected to play a role in improving the thermal stability. According to the study of Lagoudakis et al., TRPL of QW increased with temperature because of the temperature dependence on other non-radiative recombination channels ${ }^{25}$. These channels could originate from defect states, such as dislocations or interfaces. The defect would lead to a steep decrease in PLQY from an assumed $100 \%$ at $5 \mathrm{~K}$ to below $30 \%$ at room temperature. However, the efficiency of radiative energy transfer at room temperature was two orders of magnitude lower than that of NRET, $0.15 \%$ vs. $22 \%$. This underlined the importance of NRET for the efficient operation of hybrid devices.

Because NRET requires close contact between the donor and the acceptor, the interface of the LED chip is extremely demanding. The photoelectric characteristics of the rectangular-structured micro-LED caused by sidewall defects deteriorate with the shrinking of the chip size, which is more obvious for nano-structured microLEDs. Therefore, it is necessary to study the mechanism, rules, and solutions of the impact of sidewall defects on the luminescence performance of micro-LEDs. Tian et al. and Olivier et al. started several studies on this ${ }^{40,41}$. Their studies showed that sidewall damage generated during the etching of micro-LED chips contained many defects that could lead to non-radiative recombination. These defects and surface recombination mechanisms were the main reasons for the increase in leakage current and the decrease in peak external QY of microLEDs.

Using ALD technology to passivate the sidewall of 
micro-LED chips is an effective way to reduce the adverse effects of sidewall defects on chip performance ${ }^{42}$. The ALD deposition process is composed of alternate self-saturating surface adsorption chemical reactions. It is a self-limiting growth process that can strictly and precisely control the film thickness on the sub-nanometer scale, so it is especially suitable for micro-nano optical devices fabrication with a large perimeter-to-area ratio surface modification and interface control. For example, according to the study of Richters et al. ${ }^{43}$, after depositing an $\mathrm{Al}_{2} \mathrm{O}_{3}$ film on the surface of a $\mathrm{ZnO}$ nanowire structure, the separation probability of electrons and holes in the surface depletion layer was reduced, thereby improving the band gap emission of the nanowire, and effectively reducing the emission of deep energy, which is related to the surface defect of the nanowire. Nakamura et al. compared the optimization of sidewall defects made by different etching processes and deposition methods, and the sample treated by ALD and hydrofluoric acid showed excellent luminous properties ${ }^{44}$.

Recently, Wong et al. compared the optimization of sidewall defects for red micro-LED devices of different sizes $^{45}$. Figure 3(a) shows that the emission intensities decreased with smaller micro-LED devices, which resulted from the higher sidewall defect density. After ALD treatment, the luminous properties were significantly enhanced. Figure 3(b) shows the light output power characteristics of the $100 \mu \mathrm{m} \times 100 \mu \mathrm{m}$ and $20 \mu \mathrm{m} \times 20 \mu \mathrm{m}$ devices. Three groups of micro-LED devices were tested after different sidewall treatments: devices without sidewall treatments (reference), with $\mathrm{ALD} \mathrm{Al}_{2} \mathrm{O}_{3}$ sidewall treatment passivation (ALD), and with TMA/nitrogen plasma followed by $\mathrm{ALD} \mathrm{Al}_{2} \mathrm{O}_{3}$ sidewall treatment (ALD $+\mathrm{N})$. The $100 \mu \mathrm{m} \times 100 \mu \mathrm{m}$ device with sidewall treat-

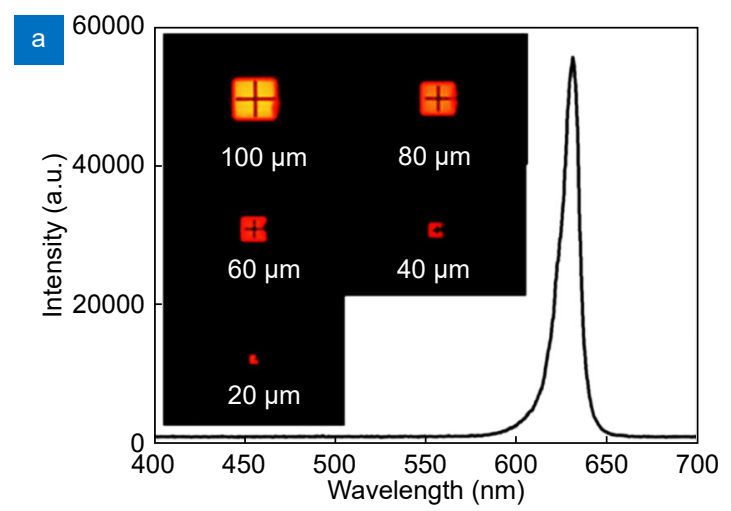

ments presented a $10 \%$ luminous enhancement compared with the related reference device. In addition, the $20 \mu \mathrm{m} \times 20 \mu \mathrm{m}$ device with sidewall treatment yielded a $150 \%$ improvement in the light output power compared with the reference device. This work showed that the ALD sidewall passivation reduces surface defects, increasing the light extraction efficiency dramatically.

In addition, the film deposited by ALD possesses good compactness, uniformity, and conformality, and has a good barrier effect on the diffusion of gas and liquid. The QD structure is usually damaged easily by high temperature and humidity. Encapsulating and protecting QDs from water and oxygen is one of the key factors to improving the stability of QDs. Using ALD to form a passivation layer on the surface of QDs is a simple and effective way. Because oxidants (such as water, ozone, hydrogen peroxide, etc.) are commonly used in ALD, which will directly react with the QDs, Kim and Martinson used an anhydrous ALD preparation process, using aluminum isopropoxide and acetic acid as precursors in $\mathrm{QDs}^{42}$. The aluminum oxide film deposited on the surface effectively improved the stability of the QDs.

$\mathrm{Wu}$ et al. reported that $\mathrm{Al}_{2} \mathrm{O}_{3}$ passivation could significantly enhance the luminous efficiency of microLEDs $^{46}$. Figure 4(a) shows the photoluminescence spectra of the packaged micro-LEDs with and without an $\mathrm{Al}_{2} \mathrm{O}_{3}$ passivation layer deposited through ALD. The emission intensities of the micro-LEDs from 430 to 515 $\mathrm{nm}$ increased by $143.7 \%$ with a passivation layer. In this paper, the increase was explained by the reduction in total internal reflection from the optical characteristics and the reduction of non-radiative recombination caused by surface trapping and defects. Furthermore, as shown in Fig. 4(b), the temperature dependent TRPL

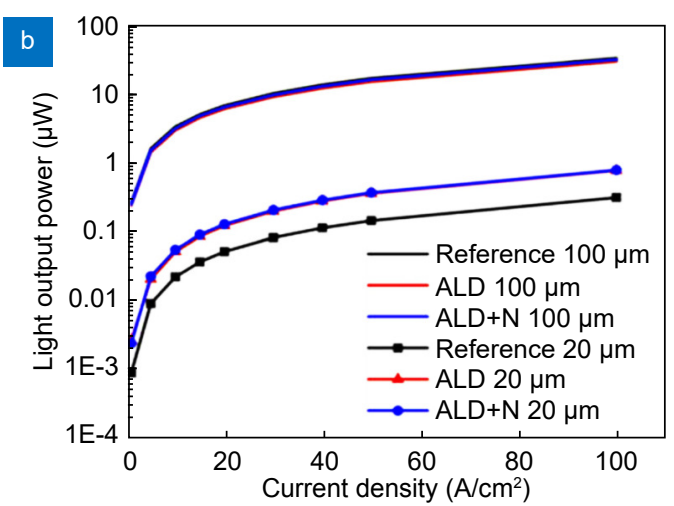

Fig. 3 | (a) Electroluminescence (EL) wavelength spectrum of a $100 \mu \mathrm{m} \times 100 \mu \mathrm{m}$ AIGalnP micro-LED and (b) light output power characteristics of $100 \mu \mathrm{m} \times 100 \mu \mathrm{m}$ and $20 \mu \mathrm{m} \times 20 \mu \mathrm{m}$ micro-LEDs with different sidewall treatments. The insets in (a) are EL images of the five micro-LED sizes at $1 \mathrm{~A} / \mathrm{cm}^{2}$. Figures reproduced with permission from ref. ${ }^{45}$, Optical Society of America. 

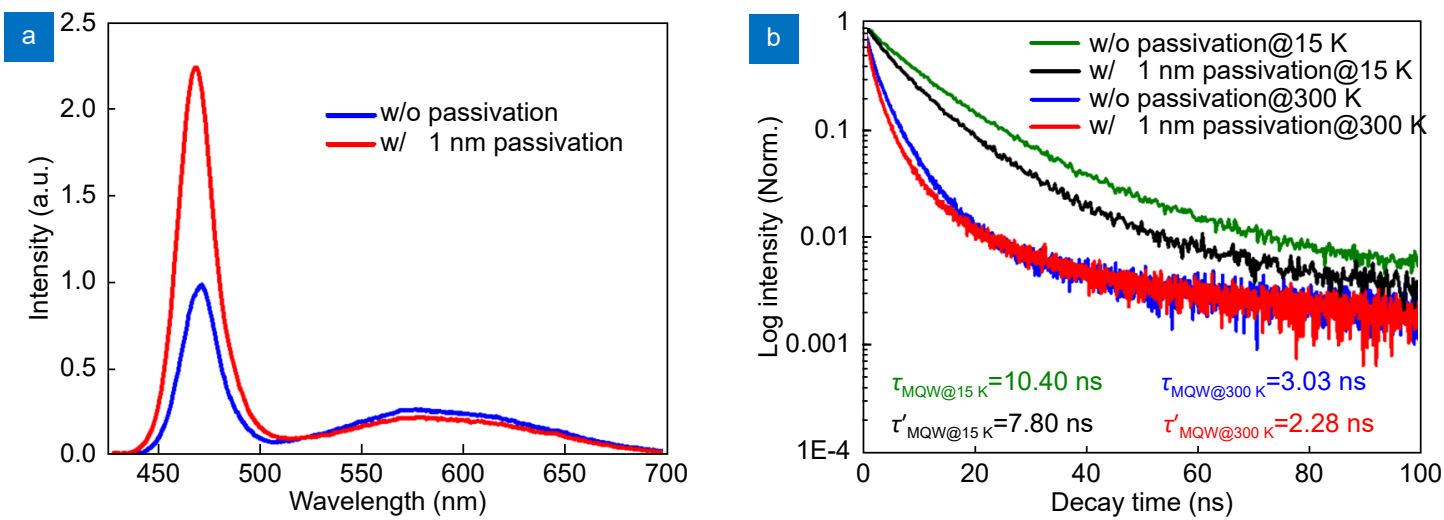

Fig. 4 | (a) PL emission spectra of micro-LEDs with and without ALD passivation. (b) Temperature-dependent time-resolved photoluminescence decay curves of micro-LEDs with and without ALD. Figures reproduced with permission from ref. ${ }^{46}$, under a Creative Commons Attribution License.

decay curves of micro-LEDs with and without ALD passivation were tested. According to the exponential decay fitting, the total recombination time of each curve was $2.28,3.03,7.80$, and $10.40 \mathrm{~ns}$, corresponding to the measurement temperatures at $15 \mathrm{~K}$ and $300 \mathrm{~K}$ for the microLED with and without passivation, respectively. The micro-LEDs with passivation decayed more quickly compared to those without passivation. This implied that the radiation combination was more efficient because of the reduction of surface defects. The surfacing would lead to a longer decay time due to the non-radiation combination even at $15 \mathrm{~K}$. In this study, the thickness and reflective index of the $\mathrm{Al}_{2} \mathrm{O}_{3}$ film deposited by ALD were $1 \mathrm{~nm}$ and 1.72 , respectively. In the future, more research on the type and thickness of the passivation layer should be conducted, which will be of great significance for the improvement of micro-LED luminous efficiency.

\section{Novel structures of micro-LED}

A shorter distance between the donor and acceptor is important for efficient NRET. According to the report of $\mathrm{H}$. Sahoo ${ }^{38}$, when the distance is less than $2 \mathrm{~nm}$, the NRET efficiency is highest, while when the distance is larger than $10 \mathrm{~nm}$, NRET fails to occur. Therefore, a large number of studies in micro-LED structures more suitable for NRET have been done ${ }^{30,47-49}$. In the following section, we review recent studies on the design of nanostructures.

\section{Nano-pillar micro-LED}

An earlier attempt has been made by utilizing the nanopillar heterostructure. $\mathrm{Xu}$ et al. reported a novel InGaN/GaN multi-QW nano-pillar LED combined with $\mathrm{CdSe} / \mathrm{ZnS} \mathrm{QDs}^{28}$. A schematic of the LED device is shown in Fig. 5(a).

The nano-pillar LEDs were fabricated by inductively coupled plasma (ICP) etching with $\mathrm{In}_{3} \mathrm{Sn}$ nanodots as the etching mask. The method was as follows. First, a $\mathrm{SiO}_{2}$ layer was deposited on the upper surface of the LED chip by plasma-enhanced chemical vapor deposition (PECVD), and an indium tin oxide (ITO) layer was deposited onto the $\mathrm{SiO}_{2}$ layer by electron beam evaporation. The samples were then immersed in a $3 \% \mathrm{HCl}$ solution to dissolve the ITO phase, leaving self-assembled $\mathrm{In}_{3} \mathrm{Sn}$ clusters on the $\mathrm{SiO}_{2}$ layer. Next, the $\mathrm{In}_{3} \mathrm{Sn}$ clusters were used as the etching mask, and the $\mathrm{SiO}_{2}$ layer was etched by a fluoride process. Finally, a patterned $\mathrm{SiO}_{2}$ hard mask was used to dry etch the LED wafer to form an InGaN/GaN nano-pillar heterostructure. By controlling the size of the $\mathrm{In}_{3} \mathrm{Sn}$ clusters and ICP etching conditions, nanopillars of $200 \mathrm{~nm}$ diameter and 50\% filling factor were successfully fabricated. The etching depth of $0.6 \mu \mathrm{m}$ was designed to achieve the n-type layer of the LED heterojunction, and the emissive QWs were exposed laterally along the sidewall of the nanopillars. Figure 5(b) shows the scanning electron microscope (SEM) image of the nano-pillar heterostructure.

After the formation of the nanopillar structure, buffer oxide etching was used to peel off the oxide hard mask, and a $\mathrm{Ti} / \mathrm{Al}(5 / 200 \mathrm{~nm})$ contact surface was formed at the bottom of the nanopillar to form an n-type electrode. To form a p-type electrode, a p-GaN surface or ITO deposited on p-type GaN was used as the electrode. Finally, a layer of colloidal CdSe/CdS core-shell QDs centered at $620 \mathrm{~nm}$ was deposited on the surface of the nanopillar by soaking the device in a solution of QDs for approximately $12 \mathrm{~h}$. Figure 5 (c) shows the SEM image of the QD- 
coated nanopillars, confirming the conformal deposition of QDs on the side wall of the nanopillar structure.

The out spectra of a nanopillar LED prior to and after QD deposition are illustrated in Fig. 6(a). The out spectra of a control sample, prepared by depositing QDs on the upper surface of a planar LED structure, are illustrated in Fig. 6(b). Comparing the two samples, the QDs contained on the nano-pillar LED possessed stronger luminescence than those contained the planar LED. The effective quantum yield (EQY) of QDs was calculated as
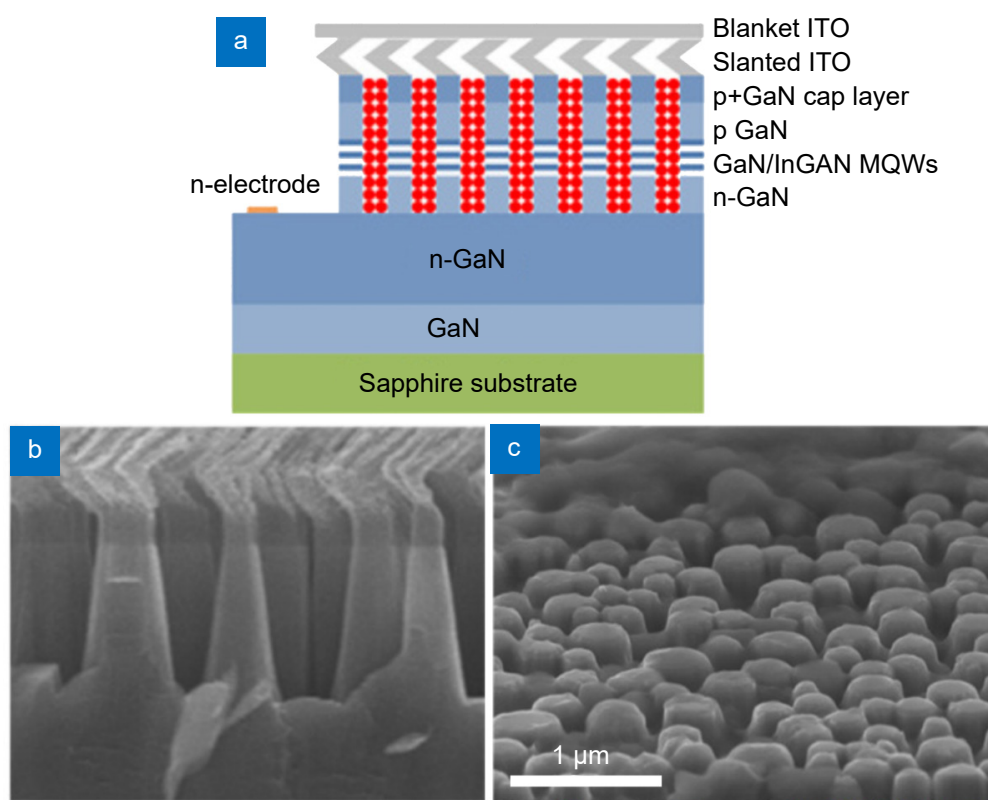

Fig. 5| (a) Schematic of the nano-pillar LED. (b) Cross-sectional SEM image of a nano-pillar LED device. (c) SEM image of the QD-coated nanopillars. Figures reproduced with permission from ref. ${ }^{28}$, Optical Society of America.
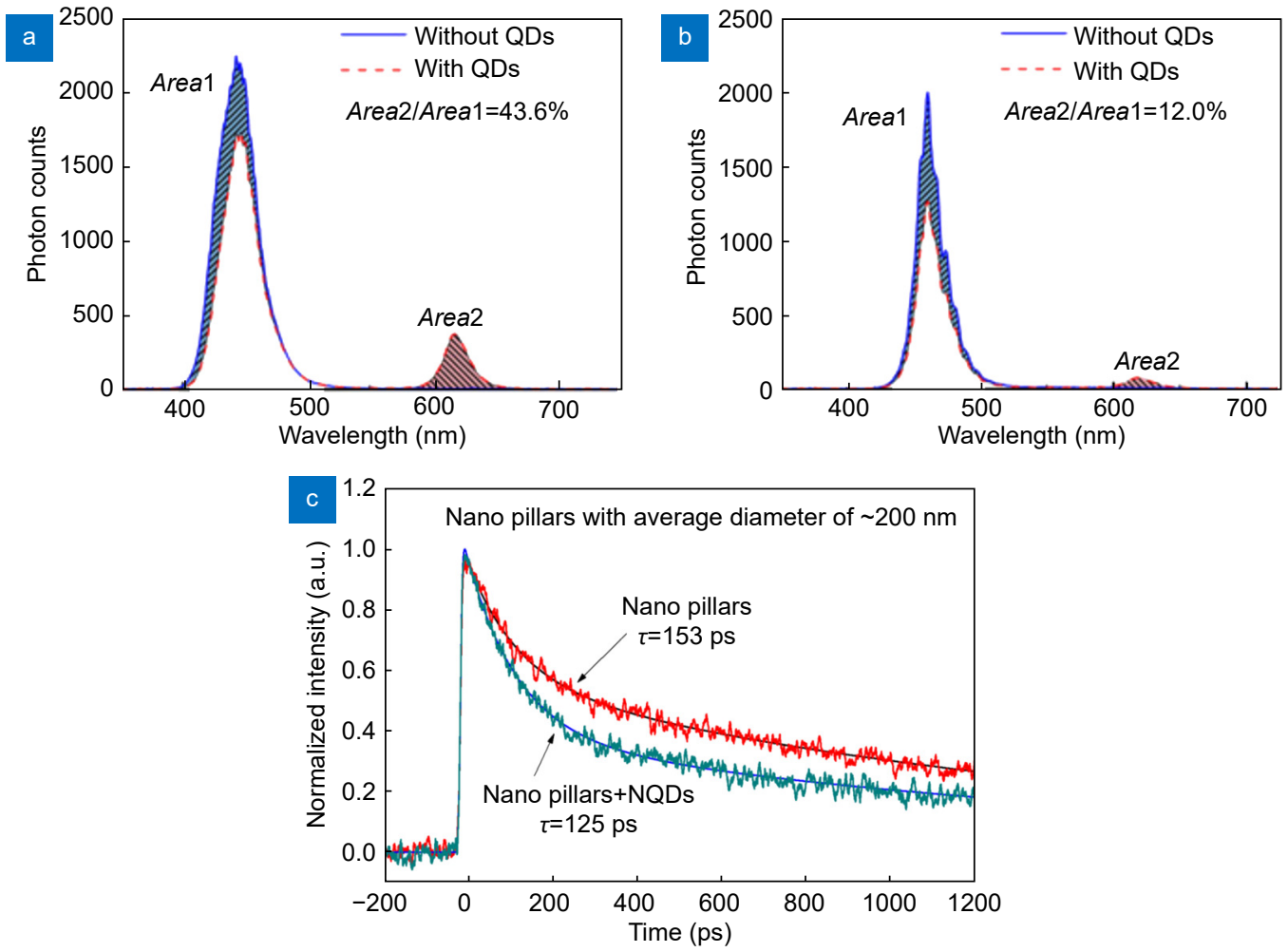

Fig. 6 | Electroluminescence spectra of the (a) nano-pillar LED and (b) planar control samples with and without QD coupling. (c) Time-resolved photoluminescence decay curves of a nano-pillar LED sample with and without QD-coating. Figures reproduced with permission from ref. ${ }^{28}$, Optical Society of America. 
43.6\% and $12.0 \%$ from the data of Fig. 6(a) and Fig. 6(b), respectively. In this study, the EQY is calculated according to the following express:

$$
E Q Y=\frac{\text { Area2 }}{\text { Area } 1},
$$

where Area1 is the reduced integral spectral area of QW after hybridization, Area 2 is the integral spectral area of QD emission.

The authors believed that the EQY of QDs on nanopillar LEDs was increased by $263 \%$. Because the EQY of QDs did not show any dependence on the substrate morphology, the significant improvement in EQY could only be explained by the substantial NRET between QDs and QWs on the side walls of the nano pillars.

Furthermore, TRPL was used to confirm the existence of NRET. As shown in Fig. 6(c), a decay life of approximately 150 ps was measured in the nano pillars without QDs, while the lifetime of QDs deposited on the surface of the nanopillars decreased to $125 \mathrm{ps}$, indicating that with the existence of QDs, the carrier decay in InGaN QW was faster. Since the deposition of QDs could not change the intrinsic carrier dynamics of InGaN QW, the faster decay meant that a relaxation channel was added to the carriers, which could only be the NRET from QW to QDs.

\section{Nano-hole micro-LED}

Nano holes are also considered to be beneficial for realizing NRET. Lagoudakis et al. carried out the opening and etching of the periodic nano-hole array on the surface of an LED chip, depositing QDs in the hole so that the QW and QDs can directly contact ${ }^{29,50}$.

Figure 7(a) shows that the device was a GaN/InGaN multi-QW LED based on a sapphire substrate. To prepare nano-hole LEDs, the following methods were utilized. A GaN nucleation layer, undoped GaN buffer layer, Si-doped $n$-GaN layer, 10 pairs of $\operatorname{In}_{0.21} \mathrm{Ga}_{0.79} \mathrm{~N} / \mathrm{GaN}$ multi-QWs with peak wavelengths of $453 \mathrm{~nm}$, and a $\mathrm{MgO}$-doped $\mathrm{p}-\mathrm{GaN}$ layer were deposited on a sapphire substrate using a bottom-up method. After the growth of the LED wafer, a transparent conductive layer ITO was formed on the wafer surface by electron beam evaporation. Nanoimprint lithography and lithography techniques were used to define nano-holes in selected mesa areas. First, a $400 \mathrm{~nm}$ thick $\mathrm{SiO}_{2}$ layer was deposited on the surface of the LED wafer by PECVD, and then a 360 $\mathrm{nm}$ thick imprinted resist layer was spin-coated onto the $\mathrm{SiO}_{2}$ layer. By placing and releasing the nanoimprint mold on the infrared layer, the nano-hole pattern of 12 photonic quasicrystals (PQCs) was transferred to the infrared layer. $\mathrm{SiO}_{2}$ nano-holes were prepared by two-step plasma reactive ion etching using $\mathrm{O}_{2}$ and $\mathrm{CHF}_{3}$

a
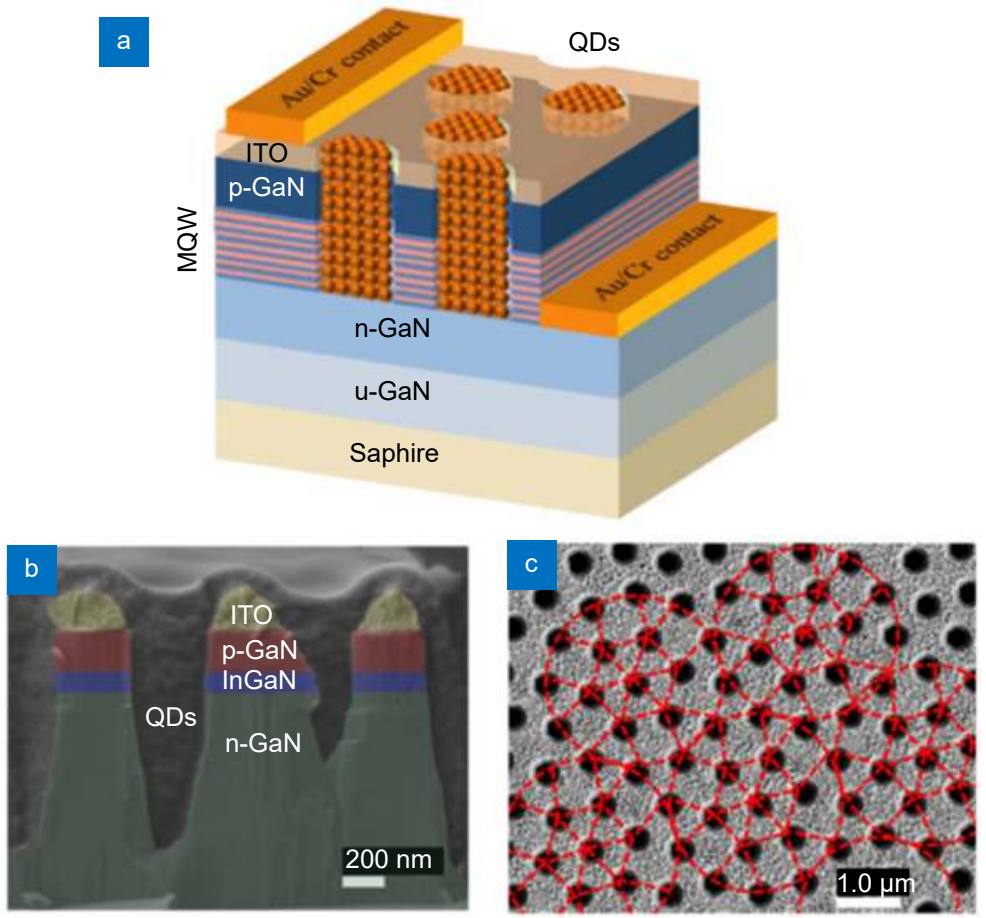

Fig. 7 | (a) Schematic representation, (b) cross-sectional, and (c) top SEM images of a photonic quasicrystal LED hybridized with QD color converters. Figures reproduced with permission from ref. ${ }^{50}$, under a Creative Commons Attribution License. 
processes. The photoresist layer was deposited on the $\mathrm{SiO}_{2}$ layer, and the selected dry etching area was determined using standard lithography technology. LED chips with a photoresist layer and a nano-patterned dielectric layer were etched by inductively coupled plasma reactive ion etching.

This highly symmetric quasicrystal has long-range ordering, short-range disorder, and semi randomness. Because of its highly symmetrical far-field beam shape and the relative increase in state density (which greatly increased the extraction of light), this geometry has been proven to be far superior to conventional PQC lattices commonly used in LED applications. The spin coating parameters were adjusted to allow the QDs to completely fill the nano-holes, as shown in Figs. 7(b) and 7(c). The LEDs were etched to induce a shunt path, and a non-radiative recombination center was formed by a bond suspended on the side wall.

The single-color converter was hybridized with QDs with an emission wavelength of $585 \mathrm{~nm}$. Figure 8(a) shows the electroluminescence (EL) spectrum of the hybrid (black solid line) and the absorption spectrum (orange dashed line) of QDs before (black solid line) and after (red solid line) hybridization. White LEDs were made of a mixture of green, orange, and red QDs. Figure 8(b) shows the EL spectra of the PQC LED and QD before and after hybridization with QDs as well as the absorption spectra of different QD colors (green, orange, and red dashed lines). There was a strong spectral over- lap between the absorption of different QDs and LEDs, which is a prerequisite for effective energy transfer between QWs and QDs. A relatively narrow (full width of half maximum is about $30 \mathrm{~nm}$ ) luminescence was observed in the mixed electroluminescence spectrum. In the case of single-color QDs and hybrid QDs, it was found that the QDs strongly quenched the EL maximum of the LED, which indicated that there was a significant energy transfer between the QW and the QDs.

Figures 8(c) and 8(d) show the CCE and EQY as a function of the injection current of the single-color LED and white LED, respectively. They were both much higher than the results of previous reports. The maximum values of CCE of the single-color LED and white LED were $66.7 \%$ and $70 \%$, respectively. Here, CCE is defined as the ratio of integral spectral area of QD emission after hybridisation to integral spectral area of blue LED before hybridization. It is believed that the increase results from the fact that the QDs were fully filled into nano-holes.

The maximum EQYs of the single-color LED and white LED were $110 \%$ and $123 \%$, respectively. Since EQY is determined by ratio of photon counts rather photon energy of emission and absorption, the value could be large than $100 \%$ during down conversion luminescence. The increase resulted from the NRET mechanism connecting the PQC LED chip to QDs, and the conclusion was proved by the TRPL curves, in which the lifetimes of the PQC LED chips decreased after combining with QDs.
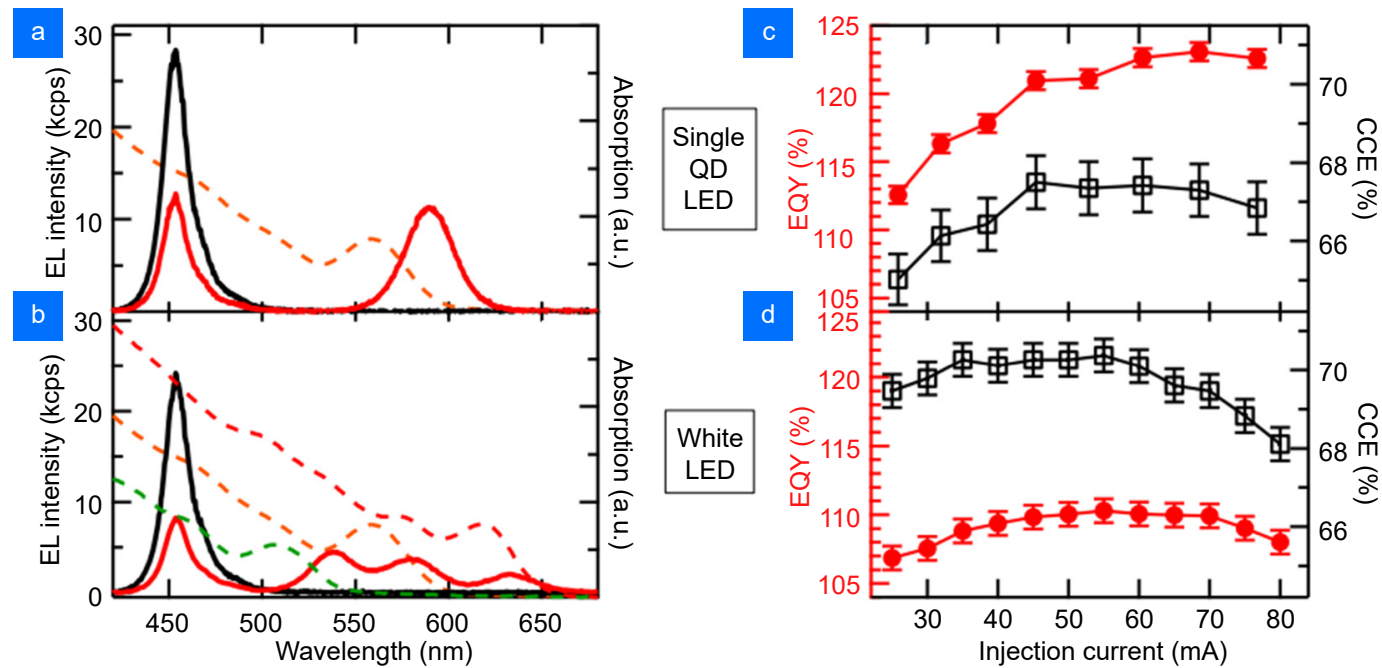

Fig. 8 | Electroluminescence spectra of a PQC LED before (black solid line) and after hybridization (red solid line) with (a) QD-585 and (b) a blend of QD-535, QD-585, and QD-630, along with the absorption spectrum of QD-585 (orange dashed line) in (a) and the absorption spectrum of QD-535 (green dashed line), QD-585 (orange dashed line), and QD-630 (red dashed line) in (b). (c, d) Effective quantum yield (red solid circles) and effective color conversion efficiency (black open squares) of a PQC LED hybridized with QD-585 in (c) and with the QD blend in (d). Figures reproduced with permission from ref. ${ }^{50}$, under a Creative Commons Attribution License. 
Zhang et al. have also prepared a new type of hybrid nano-hole periodic array/II-VI group white light LED device $^{51}$. The device used dipole coupling to enhance the NRET mechanism to achieve white light emission, exhibited high CCE and EQY, and obtained an ultra-high color rendering index.

Figure 9(a) shows a schematic diagram of the designed hybrid LED. To achieve good current diffusion, an ITO layer was deposited on the LED surface and patterned as a nano-hole. Figure 9(b) shows that the nanohole arrays with a diameter of $300 \mathrm{~nm}$ were arranged in hexagonal lattices with a lattice constant of $600 \mathrm{~nm}$. Then, CdSe/ZnS core-shell nanocrystals were filled into these nano-holes as a down-conversion medium. This structure ensured that the core/shell nanocrystals of $\mathrm{CdSe} / \mathrm{ZnS}$ were located near the active multi-QWs. Figure 9(c) shows the cross-section of the hybrid device at a depth of approximately $1.2 \mu \mathrm{m}$. The side walls of the InGaN/GaN multi-QWs were tightly surrounded by the expected nanocrystals, which was crucial for the NRET process. Figure 9(d) shows the $\mathrm{CdSe} / \mathrm{ZnS}$ core-shell nanocrystals with diameters less than $10 \mathrm{~nm}$. In this study, there was no change in the I-V curve after nanocrystals were dripped onto the h-LED, indicating that nanocrystals had no effect on the injection of electrons and holes.

In addition, the effective QY was calculated. For yel- low (546 nm) nanocrystals, the effective quantum yields were $94 \%$ and $83 \%$ in the violet and blue LEDs, respectively, which were even higher than those of the YAG: Ce phosphor. This contributed to the NRET process. Figure 10 illustrates the NRET process between the multi-QWs and nanocrystals along with the relaxation process in the hybrid LED. Because of this geometry, the excitons in multi-QWs were coupled with the $2 \mathrm{D}$ assembly of nanocrystals (NCs), which acted as a one-dimensional confined structure. As the figure shows, carriers were generated by optical pumping or current injection, and excitons were distributed in multi-QWs. If the exciton energy of multi-QWs matched with the absorption energy of NCs, these excitons could emit photons by radiation, recombine non-radiatively through defects, or undergo NRET to form the NCs components. When the energy was transferred to NCs, in-band relaxation would occur immediately, thus eliminating the resonance between the exciton and multi-QW transition and effectively preventing the reverse transfer process. Then, the relaxation excitons in NCs recombine (mainly in the form of radiation) and emit photons whose energy corresponds to the band gap of NCs.

\section{Nano-ring micro-LED}

Due to that the nano-hole structure only exposes the chip QW on the inner side of the hole wall and the
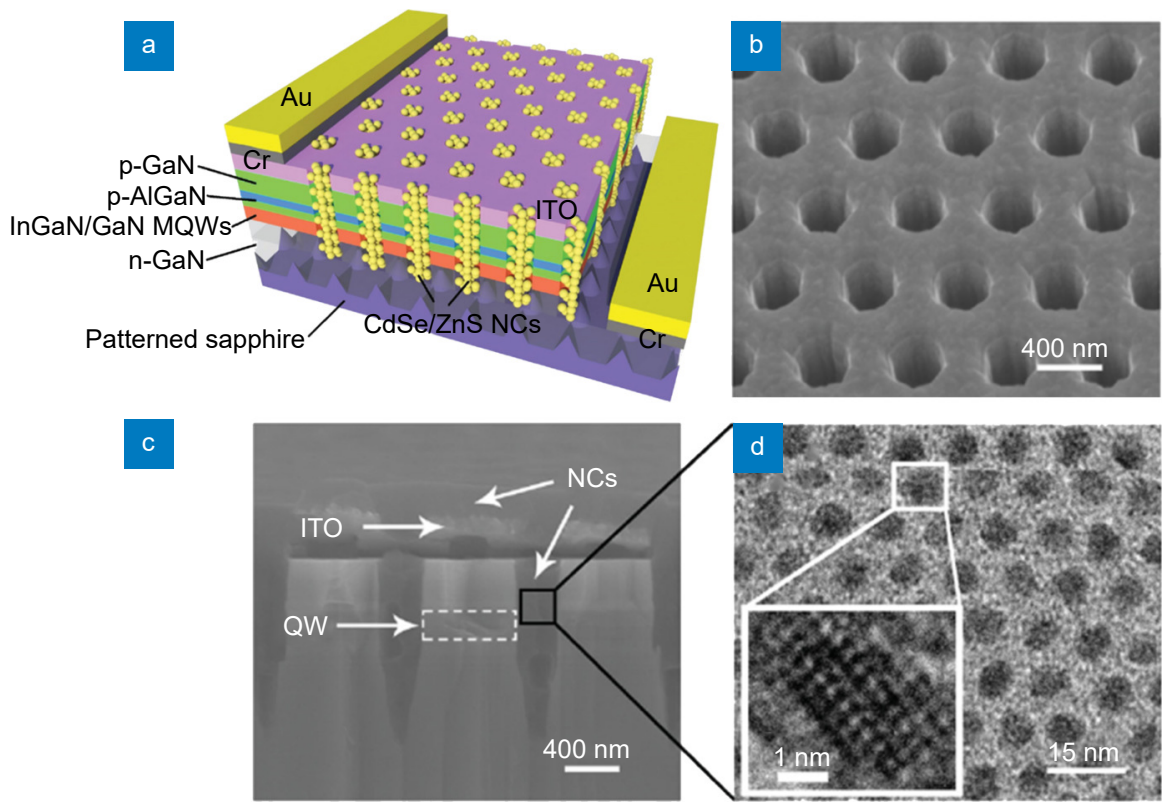

Fig. 9 | (a) Schematic diagrams of hybrid h-LED. (b) Bird's eye view SEM image of ordered nano-hole arrays in the absence of QDs with ITO current spreading layer on the surface. (c) Cross-sectional view SEM image of nano-holes filled with NCs, and (d) transmission electron microscopy (TEM) image of CdSe/ZnS core/shell QDs and a high-resolution image in the inset. Figures reproduced with permission from ref. ${ }^{51}$, John Wiley and Sons. 


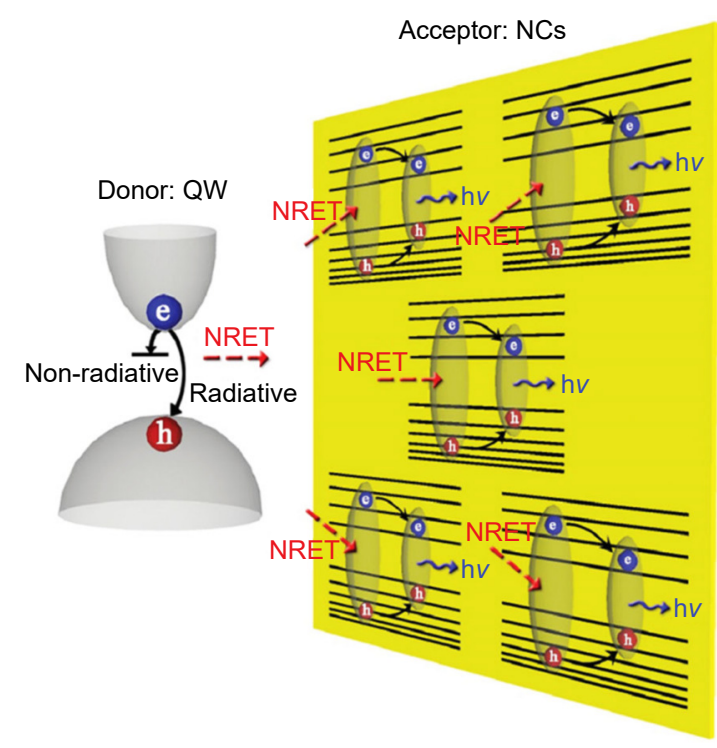

Fig. 10 | The physical schematic diagram of an NRET process. Figures reproduced with permission from ref. ${ }^{51}$, John Wiley and Sons.

nano-ring structure can expose the QW on both the inner and outer sides of the ring wall, the surface area of the chip QW in direct contact with the QDs can be increased. The proportion of NRET mechanisms in the color conversion process of QDs can also be significantly increased.

Kuo et al. used nanosphere pattern transfer technology to prepare a large area of nano-ring LED arrays with a diameter of less than $1 \mu \mathrm{m}$ on a green epitaxial wafer ${ }^{30}$. First, polystyrene nanospheres with a diameter of approximately $900 \mathrm{~nm}$ were spin-coated on the GaN LED epitaxial surface. Then, GaN-based materials were etched by inductively coupled plasma reactive ion etching (ICPRIE) to form nanopillar arrays with residual nanospheres. The diameter of the nanospheres could then be reduced by oxygen plasma treatment, and the final wall width of the nano-rings was controlled by this step. The Ni metal was deposited on the nanopillars together with the residual nanospheres using an electron beam evaporation system. An ultrasonic cleaner was used to remove the nanospheres. In the second etching process, the residual Ni protected some nanopillars and further formed a nano-ring structure. Finally, $\mathrm{Ni}$ was removed by $\mathrm{HCl}$ solution to obtain a complete nano-ring template. In this work, the nano-ring micro-LED showed color tunability with different wall widths corresponding to different internal strains. The nano-ring possessed a shorter lifetime compared to the reference sample, which meant that the nano-ring LEDs had a stronger NRET process than the reference LEDs.

Using a similar method, Wu et al. reported the use of a nano-ring micro-LED for full-color display ${ }^{46}$. In this work, the nano-ring structure was etched on a green epitaxial wafer to realize blue-emission, and then the red QDs were painted on a part of the nano-ring micro-LED as a red pixel. Figure 11 shows the process flow of the nano-ring micro-LEDs and the final device.

The manufactured QD-micro-LED was studied using SEM. Figures 12(a) and 12(b) show the SEM images with different scales and perspectives. In addition, from the

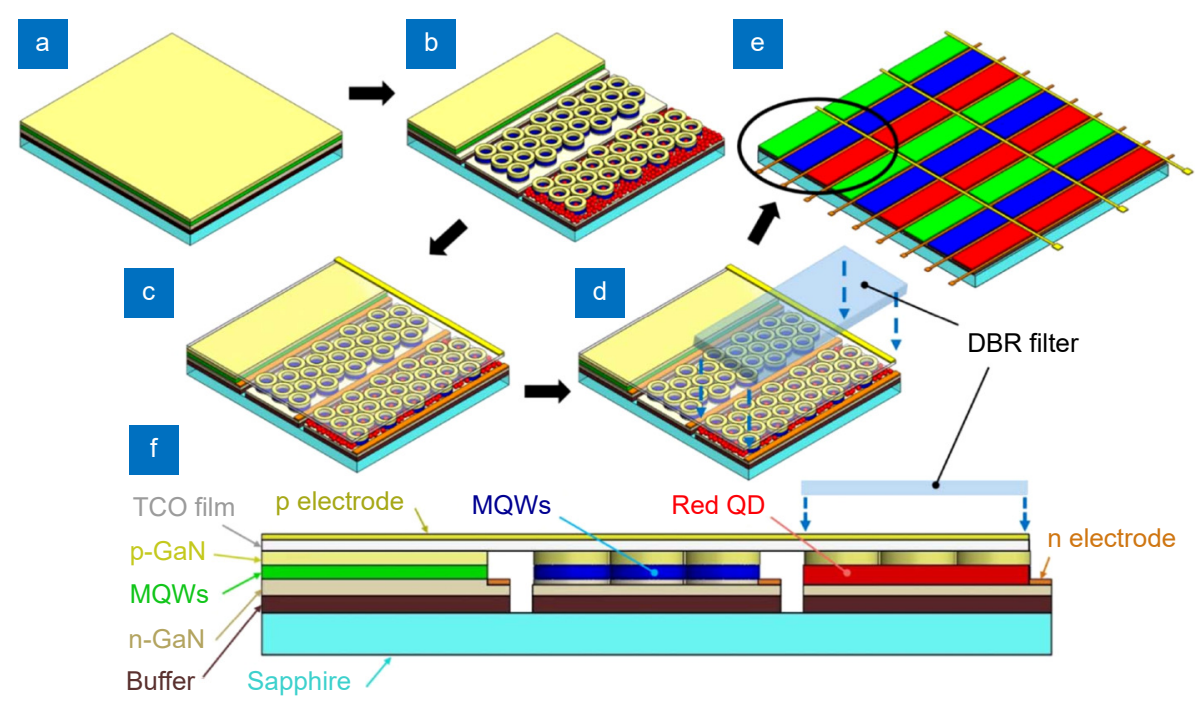

Fig. 11 | (a) Epitaxial wafer. (b) Three subpixels of a green micro-LED, a blue micro-LED, and a QD-micro-LED. (c) Deposition of transparent conducting oxide (TCO) film and P/N electrodes. (d) Covering distributed Bragg reflector (DBR) filter. (e) Full-color display panel composed of the proposed hybrid QD-micro-LED. (f) Cross-sectional view of a single RGB pixel. Figures reproduced with permission from ref. ${ }^{46}$, Chinese Laser Press. 

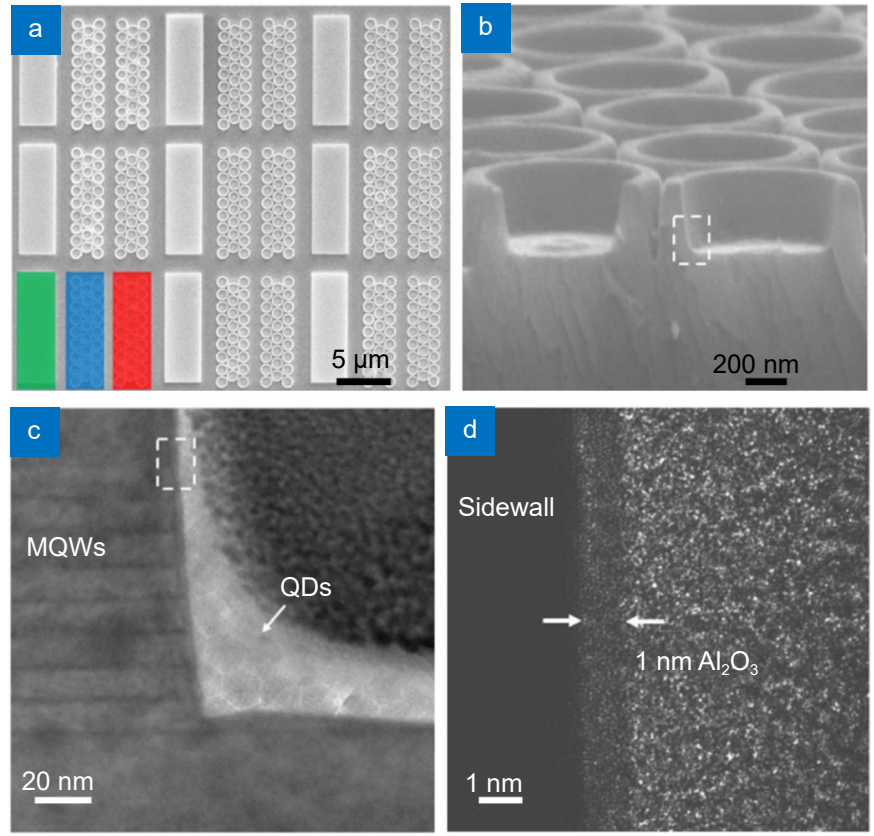

Fig. 12 | (a) SEM image of RGB pixel array. (b) SEM image of QD-micro-LED with $30^{\circ}$ tilt angle. (c) TEM image of the contact area between multi-QWs and QDs. (d) TEM image of $1 \mathrm{~nm} \mathrm{Al}_{2} \mathrm{O}_{3}$ deposited on the sidewall of an QD-micro-LED through ALD. Figures reproduced with permission from ref. ${ }^{46}$, Chinese Laser Press.

transmission electron microscopy (TEM) image in Fig. 12(c), it can be clearly observed that the sidewalls of the InGaN/GaN MQW were closely surrounded by QDs, which was essential for the NRET mechanism. Red QDs with a diameter of about $10 \mathrm{~nm}$ were also observed. In addition, it can be seen in the TEM image of Fig. 12(d) that a $1 \mathrm{~nm}$ thick $\mathrm{Al}_{2} \mathrm{O}_{3}$ layer was deposited on the sidewall of the QD-micro-LED.

In this work, the red QDs were in close contact with the blue-emission multi-QWs of nano-ring LEDs so that the red QDs could be excited efficiently. According to the wide overlap of the absorption spectrum of the red QDs and the electroluminescence spectrum of the multiQWs shown in Fig. 13(a), it was possible for NRET to exist between QDs and QWs. The TRPL curves of the QWs further confirmed NRET, as the QWs' contact with QDs possessed a shorter lifetime. The results of TRPL are displayed in Fig. 13(b). The lifetime of QWs decreased 1.12 ns after encapsulation with QDs, and the efficiency of NRET was calculated as 53.6\%. Figures 13(c) and 13(d) shows that, after structural design and spring painting, the epitaxial wafer could emit RGB light with a narrow half-width, with peak wavelengths located at $630 \mathrm{~nm}, 525$ $\mathrm{nm}$, and $467 \mathrm{~nm}$, respectively. The color gamut overlap of the NTSC space was approximately $104.8 \%$ and that of Rec. 2020 was $78.2 \%$. These results indicated that the nano-ring micro-LEDs based on the NRET mechanism was sufficient to support full-color performance in display technology.

\section{Challenges and perspectives}

As a solution, NRET plays a significant role in improving the luminous properties of QD-based micro-LEDs. According to the review, the overlap of the emission spectrum of the donor (QW) and the absorption spectrum of the acceptor (QD) as well as the sufficiently short (less than $10 \mathrm{~nm}$ ) distance between the QW and QD are important for promoting the NRET process. Nano-pillar, nano-hole, and nano-ring structures have been studied to achieve a close contact between the QW and QD, so that NRET occupies more components in the process of energy transfer. These results both improve the EQY and CCE, which will be beneficial to the improvement of display performance.

However, several problems remain to be solved. First, more stable and brighter full-color QDs are necessary to support the NRET process, which is the source of the problem. Researchers usually use the method of encapsulating organic ligands to improve the stability of QD. However, the tolerance of organic ligands to high temperature and chemical environment is poor, which limits the application of QD. Recently, the study of perovskite QD encapsulated by molecular sieve structures has attracted increasing attention ${ }^{52}$. After being 

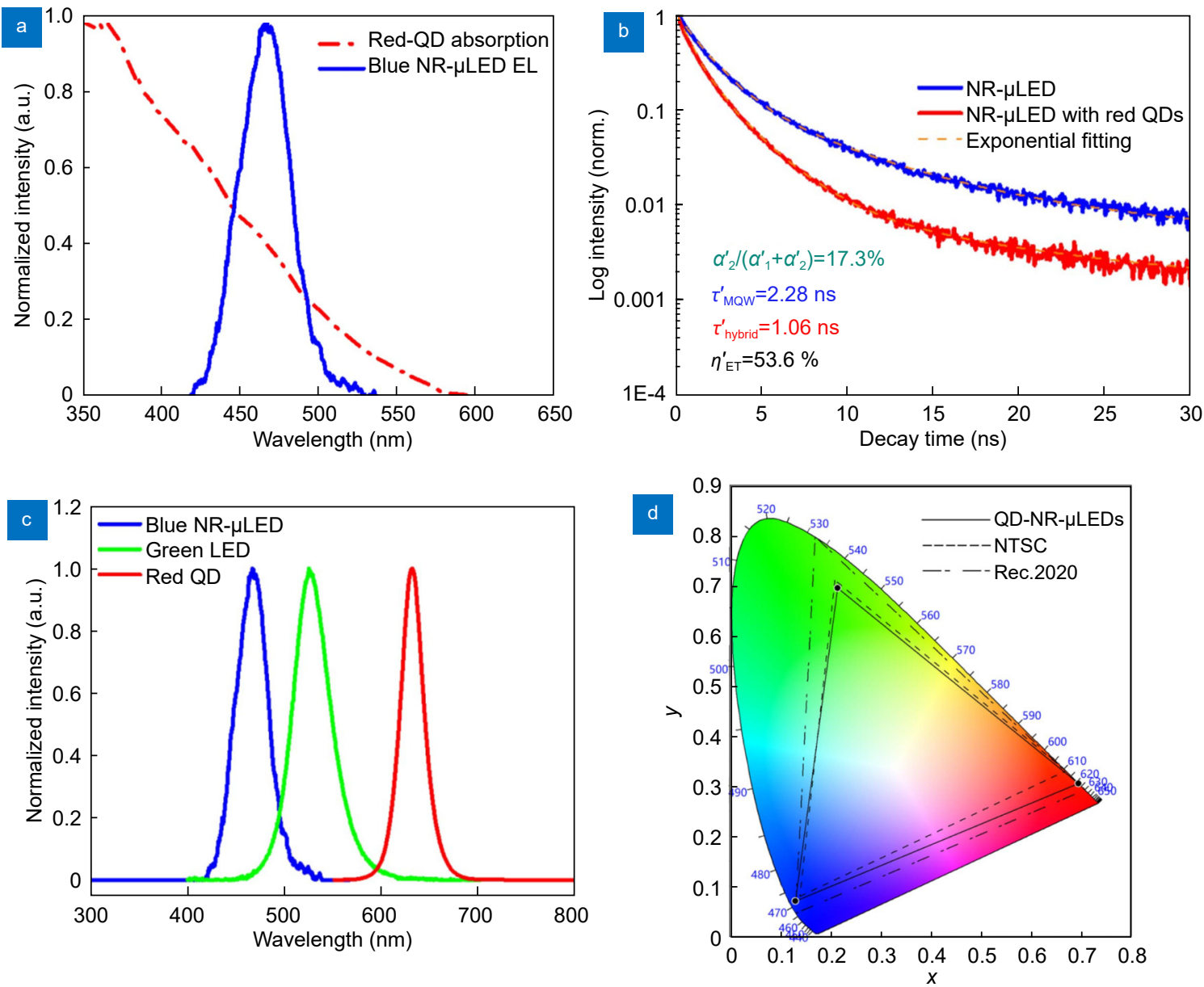

Fig. 13 | (a) Absorption spectrum of red QD and EL spectrum of blue nano-ring micro-LED. (b) TRPL curves of nano-ring micro-LED with and without red QDs. (c) Normalized EL spectra of RGB hybrid full-color micro-LED devices. (d) Color gamut of RGB hybrid full-color micro-LED device, NTSC, and Rec. 2020. Figures reproduced with permission from ref. ${ }^{46}$, Chinese Laser Press.

encapsulated by inorganic molecular sieves, perovskite QD exhibits good tolerance to water and high temperature. Although it is a promising method, the size of the coated particle is about $1 \mu \mathrm{m}$, so further smaller particle sizes are required to micro-LED. It is also undesirable that the luminescent efficiency of the particles is reduced after coating. Based on NRET, it is possible to achieve the energy transfer from the coating layer to QD, which can further improve the luminescence efficiency of the QD.

Although a variety of nanostructures have been designed, none of the current structures are perfect. For example, although nano-ring LED exposes more QWs, which increases the efficiency of NRET between QWs and QDs, the light output direction of the exposed QDs is not vertical outward but lateral, which results in low light output of the whole system and restricts the practical application. Therefore, it is necessary to design greater LED structures to improve the luminescent performance of the whole system.
With the advent of $5 \mathrm{G}$ and Sub $6 \mathrm{G}$, visible light communication is preparing for the next generation of communication technology. Visible light communication uses LED as the light source, and can be used with indoor lighting, electronic Kanban, and display as a means of wireless communication. However, the traditional large size LED light source can only reach the frequency of tens of $\mathrm{MHz}$, and there is still much room for improvement. Therefore, in addition to its application in the display, micro-LED will also have great potential in visible light communication due to its high modulation bandwidth ${ }^{12}$. Furthermore, QDs can be used in microLED to achieve a high modulation bandwidth for different wavelengths of light. It is reasonable to expect that, with the anticipated breakthroughs in the next few years, QD-based micro-LEDs possess a bright future.

\section{References}

1. Pust $P$, Schmidt $P J$, Schnick W. A revolution in lighting. Nat 
Mater 14, 454-458 (2015).

2. Reineke S. Complementary LED technologies. Nat Mater 14, 459-462 (2015).

3. Jiang HX, Jin SX, Li J, Shakya J, Lin JY. Ill-nitride blue microdisplays. Appl Phys Lett 78, 1303-1305 (2001).

4. Jin SX, Li J, Lin JY, Jiang HX. InGaN/GaN quantum well interconnected microdisk light emitting diodes. Appl Phys Lett 77, 3236-3238 (2000).

5. Jin SX, Li J, Li JZ, Lin JY, Jiang HX. GaN microdisk light emitting diodes. Appl Phys Lett 76, 631-633 (2000).

6. Wu TZ, Sher CW, Lin Y, Lee CF, Liang F et al. Mini-LED and micro-LED: promising candidates for the next generation display technology. App/ Sci 8, 1557 (2018).

7. Liu ZJ, Lin CH, Hyun BR, Sher CW, Lv ZJ et al. Micro-lightemitting diodes with quantum dots in display technology. Light Sci App/ 9, 83 (2020).

8. Yin YM, Hu ZP, Ali MU, Duan M, Gao L et al. Full-color microLED display with $\mathrm{CsPbBr} 3$ perovskite and $\mathrm{CdSe}$ quantum dots as color conversion layers. Adv Mater Technol 5, 2000251 (2020).

9. Huang YG, Hsiang EL, Deng MY, Wu ST. Mini-LED, microLED and OLED displays: present status and future perspectives. Light Sci App/ 9, 105 (2020).

10. Zhou XJ, Tian PF, Sher CW, Wu J, Liu HZ et al. Growth, transfer printing and colour conversion techniques towards full-colour micro-LED display. Prog Quant Electron 71, 100263 (2020).

11. Hong $\mathrm{YJ}$, Lee $\mathrm{CH}$, Yoon $\mathrm{A}$, Kim $\mathrm{M}$, Seong $\mathrm{HK}$ et al. Visiblecolor-tunable light-emitting diodes. Adv Mater 23, 3284-3288 (2011).

12. Chen SWH, Huang YM, Singh KJ, Hsu YC, Liou FJ et al. Fullcolor micro-LED display with high color stability using semipolar (20-21) InGaN LEDs and quantum-dot photoresist. Photonics Res 8, 630-636 (2020).

13. Gou FW, Hsiang EL, Tan GJ, Lan YF, Tsai CY et al. Tripling the optical efficiency of color-converted micro-LED displays with funnel-tube array. Crystals 9, 39 (2019).

14. Jeong CK, Park KI, Son JH, Hwang GT, Lee SH et al. Selfpowered fully-flexible light-emitting system enabled by flexible energy harvester. Energy Environ Sci 7, 4035-4043 (2014).

15. Ding K, Avrutin V, Izyumskaya N, Özgür Ü, Morkoç H. MicroLEDs, a manufacturability perspective. App/ Sci 9, 1206 (2019).

16. Wang K, Du YX, Liang J, Zhao JY, Xu FF et al. Wettabilityguided screen printing of perovskite microlaser arrays for current-driven displays. Adv Mater 32, 2001999 (2020).

17. Mei WH, Zhang ZQ, Zhang AD, Li D, Zhang XY et al. High-resolution, full-color quantum dot light-emitting diode display fabricated via photolithography approach. Nano Res 13, 2485-2491 (2020).

18. Shirasaki Y, Supran GJ, Bawendi MG, Bulović V. Emergence of colloidal quantum-dot light-emitting technologies. Nat Photonics 7, 13-23 (2013).

19. Ho SJ, Hsu HC, Yeh CW, Chen HS. Inkjet-printed salt-encapsulated quantum dot film for UV-based RGB color-converted micro-light emitting diode displays. ACS Appl Mater Interfaces 12, 33346-33351 (2020).

20. Kim T, Kim KH, Kim S, Choi SM, Jang $\mathrm{H}$ et al. Efficient and stable blue quantum dot light-emitting diode. Nature $\mathbf{5 8 6}$, 385-389 (2020)

21. Sekiguchi $H$, Kishino $K$, Kikuchi A. Emission color control from blue to red with nanocolumn diameter of InGaN/GaN nanocolumn arrays grown on same substrate. Appl Phys Lett 96, 231104 (2010).

22. Lin HY, Sher CW, Hsieh DH, Chen XY, Chen HMP et al. Optical cross-talk reduction in a quantum-dot-based full-color microlight-emitting-diode display by a lithographic-fabricated photoresist mold. Photonics Res 5, 411-416 (2017).

23. Chen HS, Hsu CK, Hong HY. InGaN-CdSe-ZnSe quantum dots white LEDs. IEEE Photon Technol Lett 18, $193-195$ (2006).

24. Zhou BZ, Liu MJ, Wen YW, Li Y, Chen R. Atomic layer deposition for quantum dots based devices. Opto-Electron Adv 3, 190043 (2020).

25. Rindermann JJ, Pozina G, Monemar B, Hultman L, Amano H et al. Dependence of resonance energy transfer on exciton dimensionality. Phys Rev Lett 107, 236805 (2011).

26. Clapp AR, Medintz IL, Mattoussi H. Förster resonance energy transfer investigations using quantum-dot fluorophores. Chemphyschem 7, 47-57 (2006).

27. Han HV, Lin HY, Lin CC, Chong WC, Li JR et al. Resonant-enhanced full-color emission of quantum-dot-based micro LED display technology. Opt Express 23, 32504-32515 (2015).

28. Zhang F, Liu J, You GJ, Zhang CF, Mohney SE et al. Nonradiative energy transfer between colloidal quantum dot-phosphors and nanopillar nitride LEDs. Opt Express 20, A333-A339 (2012).

29. Chanyawadee S, Lagoudakis PG, Harley RT, Charlton MDB, Talapin DV et al. Increased color-conversion efficiency in hybrid light-emitting diodes utilizing non-radiative energy transfer. Adv Mater 22, 602-606 (2010).

30. Wang SW, Hong KB, Tsai $\mathrm{YL}$, Teng $\mathrm{CH}$, Tzou AJ et al. Wavelength tunable InGaN/GaN nano-ring LEDs via nanosphere lithography. Sci Rep 7, 42962 (2017).

31. Itskos G, Heliotis G, Lagoudakis PG, Lupton J, Barradas NP et al. Efficient dipole-dipole coupling of Mott-Wannier and Frenkel excitons in ( $\mathrm{Ga}, \mathrm{In}) \mathrm{N}$ quantum well/polyfluorene semiconductor heterostructures. Phys Rev B 76, 035344 (2007).

32. Kos Š, Achermann M, Klimov VI, Smith DL. Different regimes of Förster-type energy transfer between an epitaxial quantum well and a proximal monolayer of semiconductor nanocrystals. Phys Rev B 71, 205309 (2005).

33. Medintz IL, Clapp AR, Melinger JS, Deschamps JR, Mattoussi $H$. A reagentless biosensing assembly based on quantum dotdonor Förster resonance energy transfer. Adv Mater 17, 2450-2455 (2005).

34. Heliotis G, Itskos G, Murray R, Dawson MD, Watson IM et al. Hybrid inorganic/organic semiconductor heterostructures with efficient non-radiative energy transfer. Adv Mater 18, 334-338 (2006).

35. Achermann M, Petruska MA, Kos S, Smith DL, Koleske DD et al. Energy-transfer pumping of semiconductor nanocrystals using an epitaxial quantum well. Nature 429, 642-646 (2004).

36. Achermann M, Petruska MA, Koleske DD, Crawford $\mathrm{MH}$, Klimov VI. Nanocrystal-based light-emitting diodes utilizing high-efficiency nonradiative energy transfer for color 
conversion. Nano Lett 6, 1396-1400 (2006).

37. Vaghasiya JV, Sonigara KK, Suresh L, Panahandeh-Fard M, Soni SS et al. Efficient power generating devices utilizing low intensity indoor lights via non-radiative energy transfer mechanism from organic ionic redox couples. Nano Energy 60, 457-466 (2019).

38. Sahoo H. Förster resonance energy transfer - a spectroscopic nanoruler: principle and applications. J Photochem Photobiol C 12, 20-30 (2011).

39. Krishnan C, Mercier T, Rahman T, Piana G, Brossard M et al. Efficient light harvesting in hybrid quantum dot-interdigitated back contact solar cells via resonant energy transfer and luminescent downshifting. Nanoscale 11, 18837-18844 (2019).

40. Tian PF, McKendry JJD, Gong Z, Guilhabert B, Watson IM et al. Size-dependent efficiency and efficiency droop of blue InGaN micro-light emitting diodes. Appl Phys Lett 101, 231110 (2012).

41. Olivier F, Daami A, Licitra C, Templier F. Shockley-read-hall and auger non-radiative recombination in GaN based LEDs: a size effect study. Appl Phys Lett 111, 022104 (2017).

42. Kim IS, Martinson ABF. Stabilizing hybrid perovskites against moisture and temperature via non-hydrolytic atomic layer deposited overlayers. J Mater Chem A 3, 20092-20096 (2015).

43. Richters JP, Voss T, Kim DS, Scholz R, Zacharias M. En-

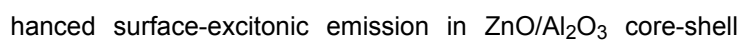
nanowires. Nanotechnology 19, 305202 (2008).

44. Wong MS, Hwang D, Alhassan Al, Lee C, Ley R et al. High efficiency of III-nitride micro-light-emitting diodes by sidewall passivation using atomic layer deposition. Opt Express 26, 21324-21331 (2018).

45. Wong MS, Kearns JA, Lee C, Smith JM, Lynsky C et al. Improved performance of AIGalnP red micro-light-emitting diodes with sidewall treatments. Opt Express 28, $5787-5793$ (2020).

46. Huang Chen SW, Shen CC, Wu TZ, Liao ZY, Chen LF et al.
Full-color monolithic hybrid quantum dot nanoring micro lightemitting diodes with improved efficiency using atomic layer deposition and nonradiative resonant energy transfer. Photonics Res 7, 416-422 (2019).

47. Liu CY, Chen TP, Kao TS, Huang JK, Kuo HC et al. Color-conversion efficiency enhancement of quantum dots via selective area nano-rods light-emitting diodes. Opt Express 24, 19978-19987 (2016).

48. Ghataora S, Smith RM, Athanasiou M, Wang T. Electrically injected hybrid organic/inorganic III-nitride white light-emitting diodes with nonradiative Förster resonance energy transfer. ACS Photonics 5, 642-647 (2018).

49. Kang JH, Li BJ, Zhao TS, Johar MA, Lin CC et al. RGB arrays for micro-light-emitting diode applications using nanoporous GaN embedded with quantum dots. ACS Appl Mater Interfaces 12, 30890-30895 (2020).

50. Krishnan C, Brossard M, Lee KY, Huang JK, Lin $\mathrm{CH}$ et al. Hybrid photonic crystal light-emitting diode renders $123 \%$ color conversion effective quantum yield. Optica 3, 503-509 (2016).

51. Zhuang Z, Guo X, Liu B, Hu FR, Li Y et al. High color rendering index hybrid III-nitride/nanocrystals white light-emitting diodes. Adv Funct Mater 26, 36-43 (2016).

52. Zhang QG, Wang B, Zheng WL, Kong L, Wan Q et al. Ceramic-like stable $\mathrm{CsPbBr}_{3}$ nanocrystals encapsulated in silica derived from molecular sieve templates. Nat Commun 11, 31 (2020).

\section{Acknowledgements}

We are grateful for financial supports from the National Natural Science Foundation of China (11904302, 61921005), Major Project of the Science and Technology in Fujian Province of China (2019HZ020013), and Major Science and Technology Projects in Xiamen of China (3502Z20191015).

\section{Competing interests}

The authors declare no competing financial interests. 